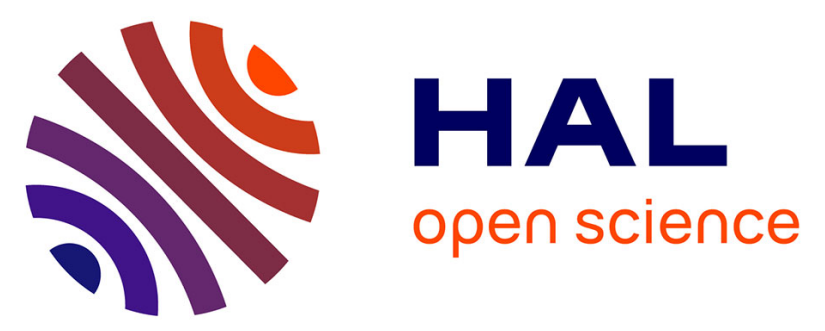

\title{
Spatio-temporal filtering with morphological operators for robust cell migration estimation in "in-vivo" images
}

David Pastor-Escuredo, Miguel Angel Luengo-Oroz, Louise Duloquin, Benoit

Lombardot, María Jesús Ledesma-Carbayo, Paul Bourgine, Nadine Peyriéras, Andrés Santos

\section{To cite this version:}

David Pastor-Escuredo, Miguel Angel Luengo-Oroz, Louise Duloquin, Benoit Lombardot, María Jesús Ledesma-Carbayo, et al.. Spatio-temporal filtering with morphological operators for robust cell migration estimation in "in-vivo" images. 9th IEEE International Symposium on Biomedical Imaging (ISBI) - From Nano to Macro, 2012, Barcelona, Spain. pp.1312-1315, 10.1109/ISBI.2012.6235804 . hal-01054803

\section{HAL Id: hal-01054803 https://hal.science/hal-01054803}

Submitted on 6 Jan 2020

HAL is a multi-disciplinary open access archive for the deposit and dissemination of scientific research documents, whether they are published or not. The documents may come from teaching and research institutions in France or abroad, or from public or private research centers.
L'archive ouverte pluridisciplinaire HAL, est destinée au dépôt et à la diffusion de documents scientifiques de niveau recherche, publiés ou non, émanant des établissements d'enseignement et de recherche français ou étrangers, des laboratoires publics ou privés. 


\title{
SPATIO-TEMPORAL FILTERING WITH MORPHOLOGICAL OPERATORS FOR ROBUST CELL MIGRATION ESTIMATION IN "IN-VIVO" IMAGES
}

\author{
D. Pastor-Escuredo $\quad$ M.A. Luengo-Oroz ${ }^{\star} \quad$ L. Duloquin ${ }^{\dagger} \quad$ B. Lombardot ${ }^{\ddagger}$ \\ $\begin{array}{lll}\text { M. Ledesma-Carbayo } & \text { P. Bourgine } & \ddagger\end{array} \quad$ N. Peyrieras $^{\dagger} \quad$ A.Santos $^{\star}$ \\ ${ }^{\star}$ Biomedical Image Technologies, ETSITelecomunicación, Universidad Politécnica de Madrid, Spain \\ ${ }^{\dagger} \mathrm{NeD}, \mathrm{CNRS}$, Institut de Neurobiologie Alfred Fessard, Gif-sur-Yvette, France \\ ${ }^{\ddagger}$ CREA-Ecole Polytechnique, Paris, France
}

\begin{abstract}
The understanding of the embryogenesis in living systems requires reliable quantitative analysis of the cell migration throughout all the stages of development. This is a major challenge of the "in-toto" reconstruction based on different modalities of "in-vivo" imaging techniques -spatio-temporal resolution and image artifacts and noise. Several methods for cell tracking are available, but expensive manual interaction -time and human resources- is always required to enforce coherence. Because of this limitation it is necessary to restrict the experiments or assume an uncontrolled error rate. Is it possible to obtain automated reliable measurements of migration? can we provide a seed for biologists to complete cell lineages efficiently? We propose a filtering technique that considers trajectories as spatio-temporal connected structures that prunes out those that might introduce noise and false positives by using multi-dimensional morphological operators.
\end{abstract}

Index Terms - Mathematical morphology, embryogenesis, cell tracking, multi-scale filtering, "in-vivo" imaging

\section{INTRODUCTION}

Developmental biology is undergoing a revolution [1][2] in the recent years thanks to the imaging techniques that allow fast acquisition and reconstruction of temporal sequences of $3 D$ images defining a $3 D+t$ data domain [3]. These microscopy techniques make possible the "in-vivo" observation of the embryogenesis for its digitalization and quantitative analysis [4][5] helping to the understanding of morphogenesis of living organisms. This is still a very open field as embryos are complex systems formed by many interconnected processes at different scales and influenced by multiple internal and external factors.

Image processing and computational analysis play a very important role in this research paradigm as automated and semi-automated methods are required to obtain quantitative measurements[6][7]. From the quantitative analysis at the cell level, the explanation of the morphology of higher level structures like tissues or organs is expected. One of the most interesting goals is to reconstruct the cell migration with accuracy, so phenomena can be contextualized in the embryogenesis dynamics.

Although, plenty of cell tracking methods exist, the task remains as a challenge due to the limitations of "in-vivo" imaging: artifacts, signal to noise ratio, spatio-temporal resolution, high density of cell populations and no distinctive features or markers for each trajectory. The general approach for cell tracking implies the segmentation of cell nuclei in $3 D$ images for the posterior time linking [8]. This strategy is very sensitive to the mentioned factors resulting in false trajectories that bring a high level of uncertainty when observing several stages of development. Additionally, mitosis detection can hardly be performed as it requires faster imaging. Therefore, cell tracking has to be supervised to enforce coherence in the estimation of the migration.

The proposed method is motivated from a practical perspective, discarding those trajectories that introduce uncertainty in the migration reconstruction. By working on the $3 D+t$ domain, we profit from integrated spatio-temporal criteria, identifying coherent trajectories and pruning out the rest. The output is a coherent subset of cell migration trajectories in $3 D+$ twindows of analysis. Besides, this subset will fasten the process of correction and completion of the whole cell lineage as the context is useful for biologists to annotate trajectories. In the next section we overview the morphological operators used to implement the method. In section 3, we describe the method in detail. Sections 4 and 5 depict the experiments and the obtained results. The last section closes with conclusions and future work.

\section{SPATIO-TEMPORAL MULTISCALE ANALYSIS}

\subsection{Mathematical morphology operators}

\subsubsection{Area and volume opening and $3 D$ granulometries}

Area opening is a connected operator based on the notion of surface area. The grey tone area opening of $f$ of size $\lambda_{a}$, denoted $\gamma_{\lambda_{a}}^{a}(f)$, is given by: $\gamma_{\lambda_{a}}^{a}(f)(\mathbf{x})=\sup \{h \leq$ $\left.f(\mathbf{x}) \mid A\left(\gamma_{\mathbf{x}}^{c}\left(X_{h}(f)\right)\right) \geq \lambda_{a}\right\}$, where $A(X)$ is the area of 
$X$. Volume opening allows us to weight the size by the intensity within the surface area: $\gamma_{\lambda_{v}}^{v}(f)(\mathbf{x})=\sup \{h \leq f(\mathbf{x})$ $\left.V\left(\gamma_{\mathbf{x}}^{c}\left(X_{h}(f)\right)\right) \geq \lambda_{v}\right\}$, being $V(X)$ the sum of the intensity within $X$. Both attribute opening/closing can be used to perform 3D granulometries of the spatial scales. Applying this analysis to "in-vivo" microscopy images, it is possible to approximately segment cell nuclei [9].

\subsubsection{Spatio-temporal granulometries using morphological reconstruction}

Previous operators extended to the $4 D$ domain allow the characterization of the spatio-temporal scales of objects in time. However, this extension brings more degrees of freedom, so it is necessary to establish spatial and temporal constraints to successfully analyze specific scales. Thus, we require a spatio-temporal analysis depending on de-correlated spatial and temporal scales $f\left(\lambda_{3} d, \delta_{t}\right)$ being the former the spatial constraint and the latter the time interval of observation $-3 D+$ $t$ domain-. This is consistent with the definition of a trajectory as a spatio-temporal connected structure -a nucleus moving along time frames-. This operator is composed of an attribute opening that works in the spatial sub-domain $-3 D$ of $3 D+t$ and a causal-anticausal $3 D+t$ morphological reconstruction by dilation [10] that ensures the temporal connectivity of the structure throughout the time interval.

To apply this operator directly in the $3 D+t$ image, a $3 D+t$ structuring element $-S E$ - has to be defined. Instead of using a $4 D-$ ball, we choose $3 D+t$ tubular structures that correspond to a $3 D$-ball that remains still in time [11]. These $S E s$ allow highlighting the spatio-temporal connectivity of trajectories to perform a $3 D+t$ segmentation "in one step".

\section{METHOD DESCRIPTION}

Cell lineage in the $3 D+t$ domain -even in different imaging modalities- can be defined as a hyper-tree composed of spatio-temporal branches -trajectories-. Due to artifacts and noise, the lineage appears as a grayscale $3 D+t$ blob where the tree branches might be interconnected or interrupted insufficient temporal resolution-. We aim at pruning the connected hyper-structures to extract the underlying hyper-tree.

We propose the method depicted in Fig.1 to achieve this pruning. The spatio-temporal operators help obtaining consistent trajectories by imposing spatial constrains and enforcing temporal connectivity: $3 D$ false positive detections do unlikely propagate consistently in time. Final refinement comes from labeling each trajectory and automatically discarding the results by analyzing their topology. However, due to memory limitations to process $3 D+t$ data and the problems to image mitosis at the right temporal scale force us to consider only $3 D+t$ windows of the hyper-tree. Also, changes through embryogenesis stages demand re-calculating the parameters. A
1D+T synthetic example with three levels of intensity -black, grey, white- is presented in Fig. 2 to illustrate the method.

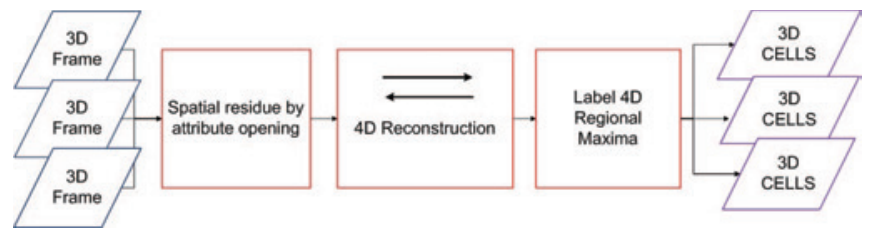

Fig. 1. Method workflow.

\subsection{Spatial filtering by attribute openings}

We filter the image with $3 D$ attribute (area/volume) openings output=opening $\lambda_{\min }$-opening $\lambda_{\max }$, imposing spatial constrains to the branches, "cutting off" at some level to match this range. These attributes mean the spatial characterization of the nuclei, so characterizing them is critical to adjust the pruning to disconnect merged trajectories (see Fig.2).
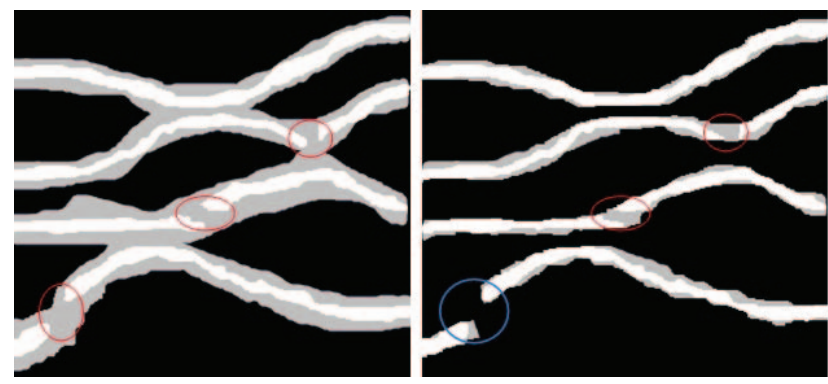

Fig. 2. Left: Three level grayscale synthetic image in $1 D+t$ domain $[t 0, t f]$. Independent trajectories (gray to white) may be interconnected at some gray level Right: Applying the $3 D$ spatial filtering by attribute openings, weakly connected branches are "disconnected" (independent trajectories or trajectories with sharper movements).

\subsection{Forward and backward reconstruction in time}

The causal and anticausal reconstructions enforce the connectivity within the interval of the $3 D+t$ window, removing the hanging or incomplete branches. Applying causal reconstruction using the proposed $S E$ and the first frame as marker, we impose connectivity forwards. Then, the resulting image is reconstructed backwards using the last frame as marker imposing connectivity backwards. Due to the reconstruction properties, the resulting image features consistent branches as $3 D+t$ regional maxima (see Fig. 3 ).

The strategy ensures the inconsistencies in the $3 D$ analysis are not propagated in time. The longer the interval, the more consistent the filtering is, but with the limits imposed by capturing many cell divisions producing disconnections and 

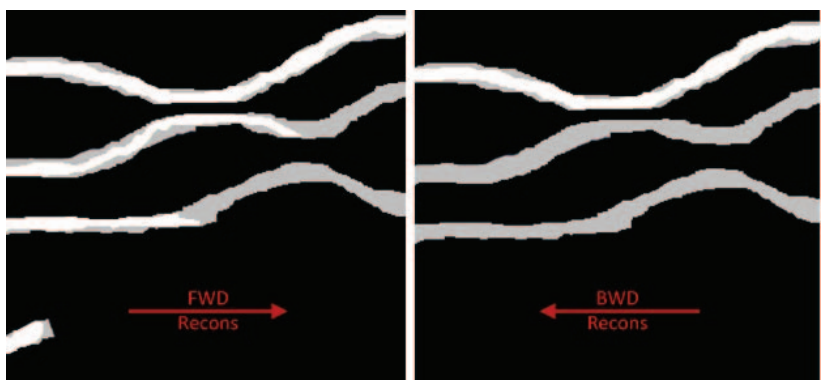

Fig. 3. Left: Anticausal reconstruction imposes the maxima in $t 0$ as $3 D+t$ maxima. Right: Causal reconstruction imposes the maxima in $t f$ as $3 D+t$ maxima. Therefore, we obtain branches as $3 D+t$ regional maxima only for connected trajectories throughout the interval.

the computational cost. Other undesired windowing effect is the pruning of trajectories getting in and out which should be not significant. Therefore, the optimal size and interval of the $3 D+t$ window are critical.

\subsection{Spatio-temporal trajectories analysis}

The resulting branches are candidate trajectories that can be labeled as $3 D+t$ objects using the $S E$. In an automated analysis, we can decompose each $3 D+t$ object in different $3 D$ sections, where each section is an hypothesis of the moving nucleus. By imposing criteria to these hypothesis we can refine the filtering obtaining a coherence-enhanced set of trajectories (see Fig.4).
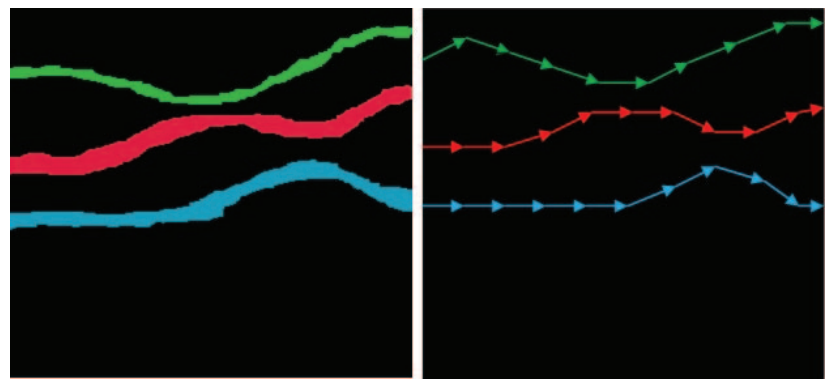

Fig. 4. Each $4 D$ regional maximum is labeled as a trajectory.

In general, the resulting $3 D+t$ objects can be classified within subsets belonging to the set $\operatorname{Trajs}(n, m)$, where $n$ is the number of $3 D$ objects in the first frame and $m$ the number of $3 D$ objects in the last frame. This means that a $3 D+t$ object starts with $n$ branches and ends up with $m$. However, as trajectories do not divide backwards, coherent trajectories can only belong to $\operatorname{Traj} s(1, m)$. Moreover, as mitosis produce two children, $m$ has to represent a coherent number of trajectories for the interval of analysis. Even so, it would be necessary to check the coherence of those sets as they might contain false mitosis. Therefore, the set containing only correct solutions a priori is $\operatorname{Traj} s(1,1)$-trajectories that did not divide-. We assume that $m$ will always reflect the divisions.

\section{EXPERIMENTS}

We have tested the method using in-vivo images of a wildtype Zebrafish embryo injected at the fertilization with the mRNA encoding fluorescent $\mathrm{H} 2 \mathrm{~B} /$ mcherry protein to stain nuclei. The dataset was acquired 4 hours post fertilization (hpf) with a time-lapse, two-photon scanning microscopy technique with $\mathrm{z}$-axis orientation from the animal pole. Data defines a $3 D+t$ domain with spatial isotropic resolution $1.37 \mu \mathrm{m}^{3}$ and a time step of 67 seconds (see Fig. 5).

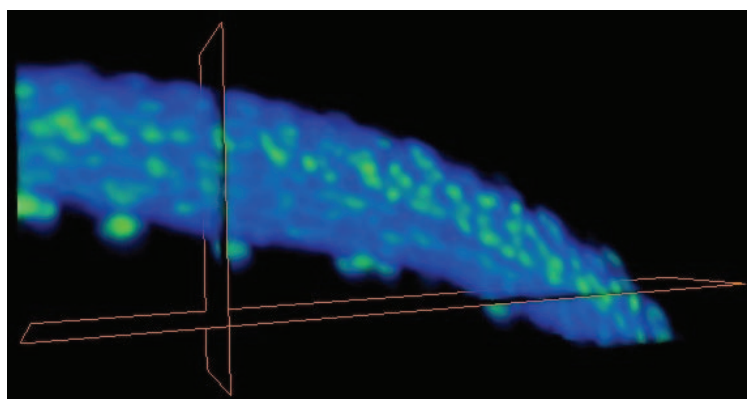

Fig. 5. Raw data after collapsing all frames into a 3D volume. It can observed the higher noise going from $3 D$ to $4 D$.

We have performed the multi-scale analysis for a $3 D+t$ window during the gastrulation of 10 time steps and containing 461 cell trajectories. The number of mitosis can be disregarded as well as the trajectories getting in and out, so that $\operatorname{Trajs}(1 / 1)$ could provide an accurate description of the cell migration. Having the parameters for this window, we could sample the embryo during this stage to get a complete migration description. The optimal parameters are the pair $\lambda_{\min }-\lambda_{\max }$ that generates largest set of $\operatorname{Trajs}(1,1)$. Parameters are obtained experimentally.

vol: $\left\{\lambda_{\min }:[800 ;+100 ; 1500]-\lambda_{\max }:[1000 ;+250 ; 5000]\right.$ (no dim) $\}$ area: $\left\{\lambda_{\min }:[10 ;+10 ; 150]-\lambda_{\max }:[50 ;+50 ; 500]\right.$ (pixels) $\}$

\section{RESULTS}

After the morphological filtering for the parameters tested, we analyze the resulting sets $\operatorname{Traj}(m, n)$ (Fig. 6). The green line represents $m=n$, under it, we find inconsistent trajectories $m>1$ or candidate mitosis $n>1$. Volume attribute proves to behave better in order to characterize the spatial dimensions of the $3 D+t$ cell lineage producing more independent trajectories than the area attribute filtering. This seems coherent as the volume attribute weights both size and intensity of the bright spots depicting nuclei in fluorescence images. 

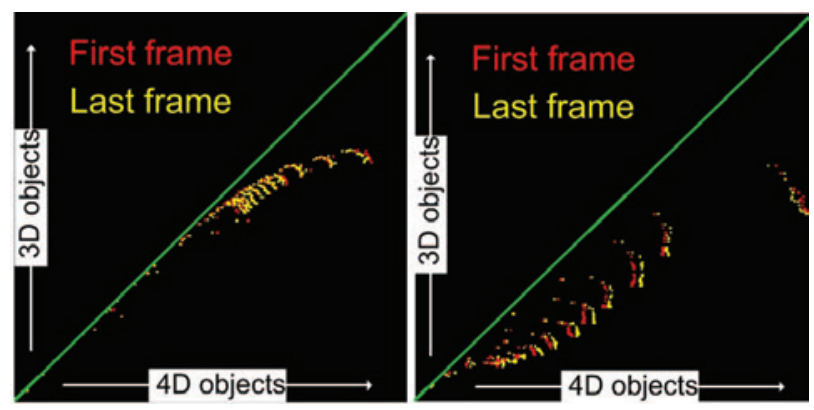

Fig. 6. Left: $n$ and $m$ values against 4D detections for the volume opening filtering. Right: same data for the area opening filtering. Greenline $=\operatorname{Traj} s(n, m)$.

Selecting the greatest $\operatorname{Trajs}(1,1)$ for the volume opening, the pair ( 800 pixels, 3000 pixels) obtains 426 trajectories $(92 \%)$. For the area opening, the best parameters (20 pixels, 75 pixels) reconstruct 364 (79\%). In Fig.7, we observe the output, forming a descriptive map of cell migration in embryogenesis suitable for quantitative analysis and to use as a reference to complete the cell lineage. These results are obtained in few hours of automated processing -varying on resources and the $3 D+t$ window size-. We assume that this output would not require supervision, in opposition to approaches that may demand up to days of human interaction, as the error rate is not as critical as knowing which trajectories are correct and which are valid.

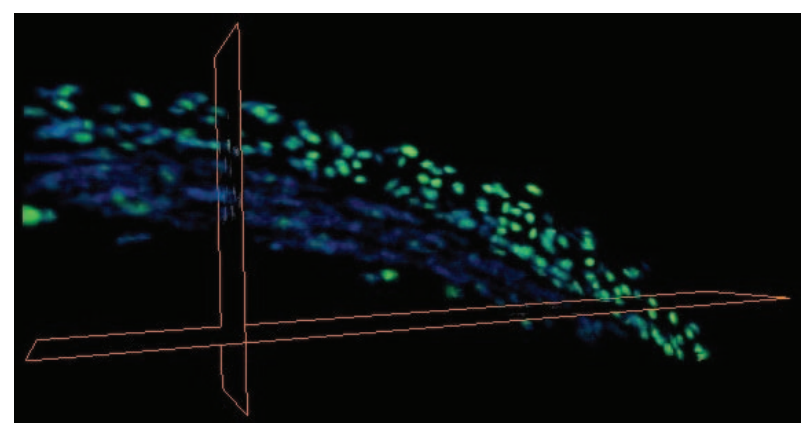

Fig. 7. Filtered data where noise has been greatly reduced keeping most part of trajectories. Each $3 D$ has been binarized and then summed and collapsed into one $3 D$ volume. Brighter trajectories (the position persists in time) stand for slow migration epithelial cells in comparison with the bottom layer of faster migrating cells during the gastrulation.

\section{DISCUSSION}

We propose a robust methodology to process data for cell migration analysis based on the selection of coherent spatiotemporal trajectories, using $3 D+t$ morphological filtering. As current "in-vivo" imaging does not reach the requirement to observe migration at the cell level, the coherent and complete reconstruction of the cell lineage is very complex and requires very time-consuming validation. The presented technique succeeds in reducing uncertainty in the results by identifying coherent trajectories inside a spatio-temporal window in an automated way. The major limitation to achieve a quasicomplete cell lineage is the scaling, as it is not capable of tracking mitosis and reconstruct more than one cell generation robustly without too restrictive time resolution. However, it is well suited for embedding in interactive tools allowing faster validation -mainly mitosis detection- and to provide an accurate estimation of migration patterns obtaining displacements fields from coherent $3 D+t$ trajectories.

\section{REFERENCES}

[1] A. Abbott, "Microscopic marvels: Seeing the system," Nature, vol. 459, no. 7247, pp. 630-631, Jun 42009.

[2] C. Vonesch, F. Aguet, J.-L. Vonesch, and M. Unser, “The colored revolution of bioimaging," IEEE Signal Processing Magazine, vol. 23, no. 3, pp. 20-31, May 2006.

[3] S. G. Megason and S. E. Fraser, "Digitizing life at the level of the cell: high-performance laser-scanning microscopy and image analysis for in toto imaging of development," Mechanisms of development, vol. 120, no. 11, pp. 1407-1420, 2003.

[4] R. Fernandez-Gonzalez, A. Munoz-Barrutia, M. H. BarcellosHoff, and C. Ortiz de Solorzano, "Quantitative in vivo microscopy: the return from the omics," Current opinion in biotechnology, vol. 17, no. 5, pp. 501-510, 2006.

[5] N. Olivier, M. A. Luengo-Oroz, L. Duloquin, E. Faure, T. Savy, I. Veilleux, X. Solinas, D. Debarre, P. Bourgine, and A. Santos, "Cell lineage reconstruction of early zebrafish embryos using label-free nonlinear microscopy," Science, vol. 329, no. 5994, pp. 967, 2010.

[6] Guy B. Blanchard and Richard J. Adams, "Measuring the multi-scale integration of mechanical forces during morphogenesis," Current opinion in genetics and development, vol. 21, no. 5, pp. 653-663, 2011.

[7] M. A. Luengo-Oroz, M. J. Ledesma-Carbayo, N. Peyriras, and A. Santos, "Image analysis for understanding embryo development: A bridge from microscopy to biological insights," Current opinion in genetics and development, vol. 21, no. 5, pp. 630-637, 2011.

[8] E. Meijering, O. Dzyubachyk, I. Smal, and W. A. van Cappellen, "Tracking in cell and developmental biology," Seminars in cell and developmental biology, vol. 20, pp. 894-902, 2009.

[9] Edmond J. Breen and Ronald Jones, "Attribute openings, thinnings, and granulometries," Computer Vision and Image Understanding, vol. 64, no. 3, pp. 377 - 389, 1996.

[10] L. Vincent, "Morphological grayscale reconstruction in image analysis: Applications and efficient algorithms," IEEE Transactions on Image Processing, vol. 2, no. 2, pp. 176-201, 1993.

[11] D. Pastor, MA Luengo-Oroz, B. Lombardot, I. Gonzalvez, L. Duloquin, T. Savy, P. Bourgine, N. Peyrieras, and A. Santos, "Cell tracking in fluorescence images of embryogenesis processes with morphological reconstruction by 4d-tubular structuring elements," in Proc. IEEE EMBC, 2009, pp. 970-973. 